\title{
Observations of Copolar Correlation Coefficient through a Bright Band at Vertical Incidence
}

\author{
D. S. ZRnić, R. Raghavan, * AND V. ChandRaseKaR** \\ NOAA / Environmental Research Laboratories, National Severe Storms Laboratory, Norman, Oklahoma
}

(Manuscript received 13 November 1992, in final form 13 July 1993)

ABSTRACT

This paper discusses an application of polarimetric measurements at vertical incidence. In particular, the correlation coefficients between linear copolar components are examined, and measurements obtained with the NSSL's and NCAR's polarimetric radars are presented. The data are from two well-defined bright bands. A sharp decrease of the correlation coefficient, confined to a height interval of a few hundred meters, marks the bottom of the bright band.

\section{Introduction}

Significant advances over the last decade have been made in improvements of rainfall estimates with polarimetric weather radars (Seliga and Bringi 1978; Ulbrich and Atlas 1984; Sachidananda and Zrnić 1987). This success is based on the fact that raindrops are oblate with the minor axis vertical so that radar echoes at two linear orthogonal polarizations (vertical and horizontal) provide additional information that can be related to drop size distribution parameters. Noting that echoes from hail are a little sensitive to polarization (Aydin et al. 1986), scientists were able to discriminate between rain and hail. These achievements capitalized on measurements of the reflectivity factor $Z$ (for one of the polarizations), the differential reflectivity $Z_{\mathrm{DR}}$, and the specific differential phase $K_{\mathrm{DP}}$ (Seliga and Bringi 1978). It is crucially important that scans be made at low elevation angles so that the anisotropy of raindrops affects the measurement. At vertical incidence the raindrops appear circular to the radar. Although hydrometeors such as graupel, hail, and snow might not look circular, they are not usually aligned in the plane of polarization. Hence, the differential reflectivity and differential phase do not contain significant information if measured at vertical incidence, but there are other quantities that can be used to discriminate between different types of hydrometeors. These

\footnotetext{
* Permanent affiliation: USRA-NASA/Marshal Space Flight Center, Huntsville, Alabama.

** Permanent affiliation: Colorado State University, Ft. Collins, Colorado.
}

Corresponding author address: Dr. Dušan S. Zrnić, National Severe Storms Laboratory, NOAA / ERL, 1313 Halley Circle, Norman, OK 73069 . are the correlation coefficient between two orthogonal copolar echo signals, the cross correlation between the main and orthogonally polarized echo, and the linear depolarization ratio (Doviak and Zrnić 1993).

The cross correlation between main and orthogonally polarized returns for circular polarizations has been measured by Hendry et al. (1976). They show that high cross correlation is a characteristic of moderate to heavy rain; low values imply the presence of ice-phase hydrometeors. Measurements of cross correlation during lightning occurrences have been made at a 3-cm wavelength by Hendry and McCormick (1976) and at $10 \mathrm{~cm}$ by Metcalf (1993), who observed a definite increase due to reorientation of crystals after the lightning flash. At horizontal incidence in rain, the cross-polar return (for horizontal or vertical polarization) is extremely small (Antar and Hendry 1985); therefore, the cross correlations between main ( $H$ or $V$ ) and orthogonally polarized ( $V$ or $H$ ) echoes do not seem to carry significant information. In the presence of randomly oriented scatterers, such as at vertical incidence, the cross correlation is zero.

The correlation coefficient between linearly polarized copolar echoes has been observed by several investigators (e.g., Bringi et al. 1991; Illingworth and Caylor 1989; Balakrishnan and Zrnic 1990). The purpose of this paper is to present and interpret the first measurements of this correlation coefficient at vertical incidence through the melting layer. These measurements were made in Florida during the Convection and Precipitation Experiment (CAPE) with NCAR's (National Center for Atmospheric Research ) CP-2 radar and in Oklahoma with NSSL's (National Severe Storms Laboratory) Cimarron radar. A low value of the correlation at the bottom of the melting layer seems to be caused by the presence of large, irregular aggregates. 


\section{Correlation coefficient}

In this section we review briefly the correlation coefficient between two linear orthogonally polarized backscattered waves. One of the linear polarizations is referred to as horizontal and the other as vertical, but that is only a carryover from horizontally scanning radars. At vertical incidence this pair can be arbitrarily oriented, yet some second-order moments [e.g., see Zrnić (1991) for relations between second-order moments and polarization variables] of the echo signals might have discriminating properties with respect to the type of echoing hydrometeors.

The correlation coefficient is defined by

$$
\rho_{h v}(0)=\frac{\left\langle s_{v v} s_{h h}^{*}\right\rangle}{\left[\left\langle\left|s_{h h}\right|^{2}\right\rangle^{1 / 2}\left\langle\left|s_{v v}\right|^{2}\right\rangle^{1 / 2}\right]} .
$$

Here $s_{i j}$ is the backscattering matrix element of a hydrometeor (McCormick and Hendry 1985). The first subscript refers to the polarization of the backscattered field ( $h$ or $v$ ) and the second to the polarization of the incident field. But in $\rho_{h v}$, the meaning of subscripts is different; each denotes the copolar transmitted and received polarization. The brackets are statistical expectations expressed in terms of the distribution of the hydrometeor's properties (i.e., equivalent volume diameter, shape, canting angle, etc.).

Throughout this paper we consider only the magnitude of the correlation coefficient. NSSL's Cimarron radar provides $\left\{\rho_{h v}(0) \mid\right.$ in real time and at 768 range locations spaced $150 \mathrm{~m}$ apart (Zahrai and Zrnić 1993), whereas the CP-2 radar, at the time of CAPE, processed data at 200 range locations spaced $200 \mathrm{~m}$ apart. The radars transmit an alternating sequence of $H$ and $V$ polarizations, and a procedure suggested by Balakrishnan and Zrnic $(1990)$ is used to obtain $\left|\rho_{h v}(0)\right|$. This procedure is based on two assumptions. First, some a priori model for the power spectral shape such as Gaussian is needed (Doviak and Zrnić 1993). Second, the correlation at lag $(2 m+1) T_{s}$ (where $T_{s}$ is the pulse repetition time) is assumed to contain independent contributions from Doppler spectral broadening and $\rho_{h v}(0)$, so that it can be expressed as a product $\rho(2 m$ $+1) \rho_{h v}(0)$ (Sachidananda and Zrnić 1986). At lag $2 m$ the correlation depends only on spectral broadening due to random motion of scatterers and at lag $2 T_{s}$ it is given by

$$
\hat{\rho}(2)=\frac{\sum\left(H_{2 i} H_{2 i+2}^{*}+V_{2 i+1} V_{2 i+3}^{*}\right)}{(M+1)\left(\hat{P}_{h}+\hat{P}_{v}\right)},
$$

where $H_{2 i}$ and $V_{2 i+1}$ are two successive complex echo samples, $P_{h}$ and $P_{v}$ are the mean sample powers at $H$ and $V$ polarizations, $M$ is the number of $H$ or $V$ sample pairs, and the circumflex denotes estimates. An estimate of $\rho_{h v}(1)$ is obtained as

$$
\left|\hat{\rho}_{h v}(1)\right|=\frac{\left|\hat{R}_{a}\right|+\left|\hat{R}_{b}\right|}{2\left(\hat{P}_{h} \hat{P}_{v}\right)^{1 / 2}},
$$

where $R_{a}$ is the autocorrelation between successive $H$ and $V$ polarized echoes and $R_{b}$ is the autocorrelation between $V$ and $H$ polarized echoes.

The correlation coefficient is computed directly from Eqs. (2) and (3) as

$$
\left|\hat{\rho}_{h v}(0)\right|=\left|\frac{\hat{\rho}_{h v}(1)}{\hat{\rho}(1)}\right|=\frac{\left|\hat{\rho}_{h v}(1)\right|}{|\hat{\rho}(2)|^{1 / 4}},
$$

because the assumption of Gaussian spectral shape permits equating $|\rho(1)|$ to $|\rho(2)|^{1 / 4}$.

The powers $P_{h}$ and $P_{v}$ in (2) and (3) contain both signal and noise; therefore, a correction must be incorporated to eliminate the noise bias. The following multiplicative correction of either prevents generation of negative powers:

$$
P_{c}=\frac{P(S / N)}{(S / N+1)}
$$

where $P_{c}$ stands for corrected power, $S$ is the signal power, and $N$ is the white noise power.

Equation (4) without noise correction (i.e., $S / N \gg 1$ ) is used in the programmable signal processors on the Cimarron and the CP-2 radars, but the correction is applied after the fact to the recorded $\left|\rho_{h v}(0)\right|$ data from the Cimarron radar and is not applied to the data from the $\mathrm{CP}-2$ radar. Although the estimate of the receiver noise is very accurate, other contributions such as quantization noise and external interferences can still bias the correlation coefficient.

Practical details pertinent to errors in the measurements are summarized in Table 1 . The errors were obtained from graphs in Doviak and Zrnić (1993) and Liu et al. (1993).

There are several meteorological factors that influence $\left|\rho_{h v}(0)\right|$. These are related to apparent shape, size, and differential phase shift upon scattering, all of which often occur simultaneously in nature. The differential phase shift upon scattering is significant for Mie scatterers only. All the other listed effects are significant for both Mie and Rayleigh scatterers. Also, in mixtures of two types of hydrometeors the correlation is lower than in either one of the hydrometeor populations (Jameson 1989a).

Decorrelation occurs if the two orthogonal backscattered fields do not vary in unison; that is, there

TABLE 1. Errors of measurement for signal-to-noise ratios of greater than $20 \mathrm{~dB}$ and Doppler spectrum widths between 1 and $4 \mathrm{~m} \mathrm{~s}^{-1}$ (SD-standard deviation).

\begin{tabular}{lcc}
\hline & Cimarron & CP-2 \\
\hline Dwell time $(\mathrm{ms})$ & 197 & 128 \\
$\mathrm{SD}(Z)(\mathrm{dB})$ & 1 & 1.5 \\
$\mathrm{SD}(v)\left(\mathrm{m} \mathrm{s}^{-1}\right)$ & 0.5 & 0.5 \\
$\mathrm{SD}\left(\sigma_{v}\right)\left(\mathrm{m} \mathrm{s}^{-1}\right)$ & 0.5 & \\
$\mathrm{SD}\left(\left|\rho_{h v}(0)\right|\right)$ & 0.014 & 0.02 \\
$\mathrm{SD}(\mathrm{LDR})(\mathrm{dB})$ & & 2 \\
\hline
\end{tabular}


(a)

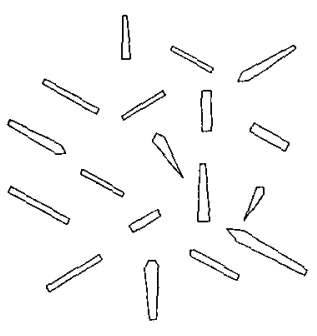

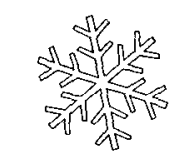

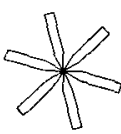

\section{5}
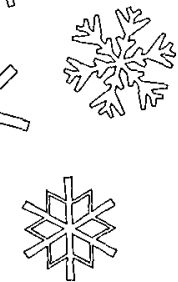

Fig. 1. (a) Needles and (b) stellar crystals randomly oriented in the plane of polarization.

must be a change of the net effective backscattering properties at horizontal and vertical polarizations in the resolution volume. This can occur if the changes of the two backscattered fields from particle to particle are not proportional to each other and there is reorientation and/or replenishment of the particles during dwell time. Obviously, oriented needles would have the same change in both horizontal and vertical backscattered fields from crystal to crystal, and the correlation would be unity. But if the crystals are randomly oriented this would not happen; hence, $\left|\rho_{h v}(0)\right|$ would decrease because the random orientation would evolve due to turbulence and replenishment of crystals. Figure 1 illustrates a random distribution of ice needles and snow flakes. These crystals are in the plane of polarization (i.e., the horizontal axis is left to right and the vertical is bottom to top) and would appear as shown at one instant (one transmitted pulse) to a radar beam at vertical incidence. The instantaneous horizontally polarized backscattered electric field from the crystals in Fig. la would be proportional to the sum of the contributions by each crystal; for Rayleigh scatterers each contribution is approximately proportional to the product of the electric field component along the needle with the length cubed projected onto the horizontal axis. Analogous results hold for the vertically polarized fields. Because the crystal configuration evolves either due to orientation changes or replenishment in and exodus from the resolution volume, the net $H$ and $V$ fields would change differently from pulse to pulse, thus reducing the correlation. Identical mechanisms cause the decorrelation of fields from symmetric ice crystals as in Fig. $1 \mathrm{~b}$ because the stellar structures are randomly oriented in the polarization plane. The decrease in correlation is more pronounced for large, irregular hydrometeors. Furthermore, as shown in Fig. 2, wetness enhances the decorrelation.

The fields also change differently if there is a variation of differential phase shift upon scattering from hydrometeor to hydrometeor. Such variations can occur if scattering is in the Mie regime; Zrnić et al. (1993) show evidence of significant differential phase shift at the bottom of the melting layer (observed with a hor-

izontally pointing beam) and attribute a portion of the decrease in $\left|\rho_{h v}(0)\right|$ to this effect.

There have been no measurements of the correlation coefficient at vertical incidence; for horizontal incidence and in a rain medium, theory predicts that $\left|\rho_{h v}(0)\right|$ will be very close to unity (Sachidananda and Zrnić 1985). Observations with a horizontally pointing beam show that the distribution of shapes (i.e., eccentricity of oblate spheroids due to dependence on size) decreases the correlation; for example, Balakrishnan and Zrnić ( 1990 ) report a mean value of 0.98 , whereas Illingworth and Caylor (1991) measured, with a high resolution (beamwidth $0.25^{\circ}$ ) radar, values between 0.985 and 0.995 . The physical reason for this dependence is that changes in reflectivities at horizontal and vertical polarization are not equal for the same increment in size/volume of hydrometeors (Jameson 1987; Jameson and Dave 1988). The distribution of sizes can affect the correlation at vertical incidence if there is significant canting so that the drop projection on a horizontal plane looks ellipsoidal.

The variety of hydrometeor shapes in the melting layer is large, but for computing the correlation coefficient (measured at vertical incidence) two basic models-prolate spheroids and distorted spheres-can be used. In this manner, qualitative if not quantitative description of bulk hydrometeor properties can be obtained. Randomly oriented spheroids in the plane of polarization are a reasonable model for elongated hydrometeors. This model can also be used to calculate the correlation coefficient of irregular hydrometeors. Intuitively, it is expected that distorted spheres should model a random distribution of rugged hydrometeors with shapes that are generally circular. From the value of the correlation coefficient alone it is not possible to deduce if backscattering hydrometeors are elongated but randomly oriented as in Fig. 1a or if the shapes are rugged (symmetric as in Fig. $1 \mathrm{~b}$ or irregular). Nev-

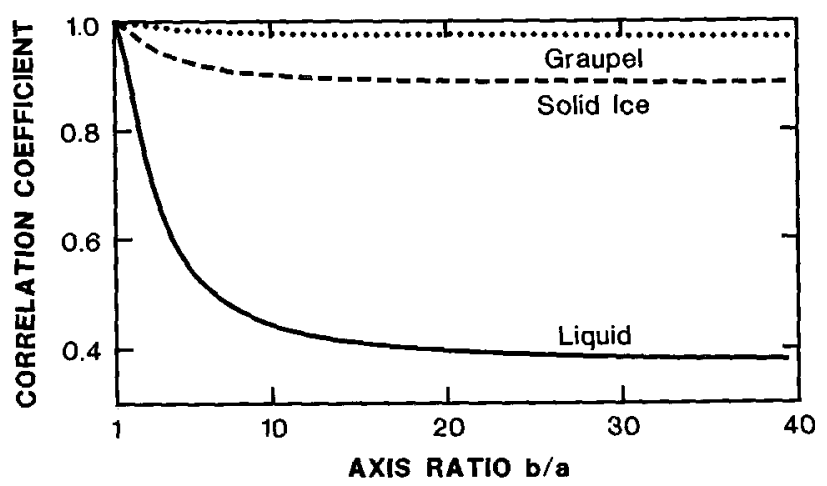

Fig. 2. Correlation coefficient for prolate spheroids with the major (larger) axis $b$ randomly oriented in the plane of polarization versus major-to-minor axis ratio $(b / a)$. Dotted curve is for ice with a density of graupel ( refractive index $m=1.372$ ), dashed curve is for solid ice $(m=1.78+j 0.007)$, and full curve is for predominately wet spheroids $(m=9.0585+j 1.3421)$; the frequency is $2.88 \mathrm{GHz}$. 
ertheless these simple models can provide a plausible explanation for otherwise speculative conclusions concerning bulk hydrometeor properties. Two model examples illustrate the values that the correlation coefficient might have at vertical incidence.

Prolate spheroids. Closed-form solutions for scattering by prolate spheroids in the Rayleigh regime are available (Doviak and Zrnić 1993). For ice spheroids and a $10-\mathrm{cm}$ wavelength, computations indicate that the correlation reduces to about 0.9 (Fig. 2$) ;\left|\rho_{h v}(0)\right|$ for wet spheroids is much smaller. Spheroids with densities of graupel also reduce the correlation but only to about 0.97 . In the Mie regime of scattering the correlation should be smaller.

Distorted spheres. Irregular shapes of hydrometeors contribute to a noticeable decrease of $\left|\rho_{h v}(0)\right|$. For distortions independent in horizontal and vertical directions and small compared to the diameter of the hydrometeor, $\left|\rho_{h v}(0)\right|$ is given in the Rayleigh limit by an expression that relates the rms value of the distortions, $\sigma_{D}$, to the equivalent diameter of the scatterer $D_{e}$ [Eq. (11) and Fig. 7 in Balakrishnan and Zrnić (1990)]. This expression is an approximation and has no dependence on the refractive index but is expected to be better for wet hydrometeors; the result indicates that for $\sigma_{D} / D_{e}>0.15$ the correlation is less than 0.8 . Similar or larger effects are expected from distorted Mie scatterers.

Correlation coefficient for randomly oriented symmetric particles such as prolate spheroids can be related to the linear depolarization ratio LDR. Jameson (1989b) used Eq. (17) from Jameson and Dave (1988) to show that the minimum the correlation coefficient can achieve is given by

$$
\left|\rho_{h v}(0)\right|_{\min }=1-2 \times 10^{(0.1 \mathrm{LDR})} .
$$

We use this expression to help interpret the data from CAPE in the next section.

\section{Measurements}

Sample measurements in two precipitation events are presented to illustrate the potential utility of the correlation coefficient to identify regions of mixedphase hydrometeors at vertical incidence. Under ideal conditions (without noise and/or artifacts) the mean correlation coefficient from pure rain at vertical incidence would be larger than 0.99 (Sachidananda and Zrnić 1986). This is because, at vertical incidence, shape variation with size is not apparent and the decrease in correlation would be due to secondary effects such as drop oscillations, coalescence, and breakup, etc. Fairly large values of $\left|\rho_{h v}(0)\right|$ are also expected from most frozen precipitation, but a decrease could occur in the presence of hail, large wet aggregates, and mixed-phase precipitation (Balakrishnan and Zrnić 1990).
Values of reflectivity factor $Z_{10 H}$ (10-cm-wavelength horizontal polarization), Doppler velocity $(10-\mathrm{cm}$ wavelength ), LDR (3-cm wavelength), and correlation coefficient $\left|\rho_{h v}(0)\right|(10-\mathrm{cm}$ wavelength $)$ obtained from precipitation in Florida with the CP-2 radar are plotted in Fig. 3. Figures 4 and 5 feature data collected with the 10-cm-wavelength radar (Cimarron) located adjacent to the Page airport $40 \mathrm{~km}$ northwest of Norman, Oklahoma.

Vertical profiles in Fig. 3 were collected in a stratiform region trailing a large convective storm. Liquid water precipitation is located between ground and the $3-\mathrm{km}$ height. The rain region is separated from the low density ice aloft by a melting layer indicated by a radar bright band. The sharpest gradients in Doppler velocity coincide with the minima of $\left|\rho_{h v}(0)\right|$, suggesting that large aggregates in the final stage of melting are responsible for the decrease. The $\left\{\rho_{h v}(0) \mid\right.$ minima are centered on the maxima of the LDR but the notches of these minima are narrower than the widths of the maxima. This might be attributed to the difference in wavelengths. The LDR obtained with a $3-\mathrm{cm}$ wavelength is sensitive to smaller irregular particles that for this wavelength can be in the Mie region of scattering. At a $10-\mathrm{cm}$ wavelength the Mie scattering occurs for larger sizes; thus, if Mie scattering is partially responsible for the $\left|\rho_{h v}(0)\right|$ minima, the notches will be narrower.

On average the correlation coefficient minimum is about 0.82 (Fig. 3d), and the maximum of LDR is about $-10 \mathrm{~dB}$. Substituting this LDR in (6) we obtain a minimum $\left|\rho_{h v}(0)\right|$ of 0.8 . These values suggest that much of the decorrelation found at vertical incidence can be explained by random orientation of distorted scatterers that can be modeled as prolate spheroids.

Data from a stratiform region of a mesoscale convective system are presented in Fig. 4. The profile of $Z$ depicts a typical bright band (Battan 1973); a layer of reflectivity above $37 \mathrm{dBZ}$ is between 2.1 and 2.4 $\mathrm{km}$. It appears that the $0^{\circ} \mathrm{C}$ isotherm had been lowered either by precipitation or change in the environment; a sounding $40 \mathrm{~km}$ to the southeast and $2 \mathrm{~h}$ earlier indicated the freezing level was at $3.4 \mathrm{~km}$ above ground and there was a $400-\mathrm{m}$-deep isothermal layer (Willis and Haymsfield 1989) extending down to $3 \mathrm{~km}$. The dip in correlation occurs at the bottom of the reflectivity layer $(1.95 \mathrm{~km})$. Similar dips of the correlation coefficient at the bottom of the melting layer were previously observed with the antenna beam at low elevation angles; these were attributed to the variety of sizes and shapes and rapid changes of the differential phase shift upon scattering (Illingworth and Caylor 1991; Zrnić et al. 1993). Just before collapsing into drops, large aggregates are most irregular and become wet; moreover, at that time there is also a substantial amount of drops present in the resolution volume: both these effects tend to decrease the correlation. 

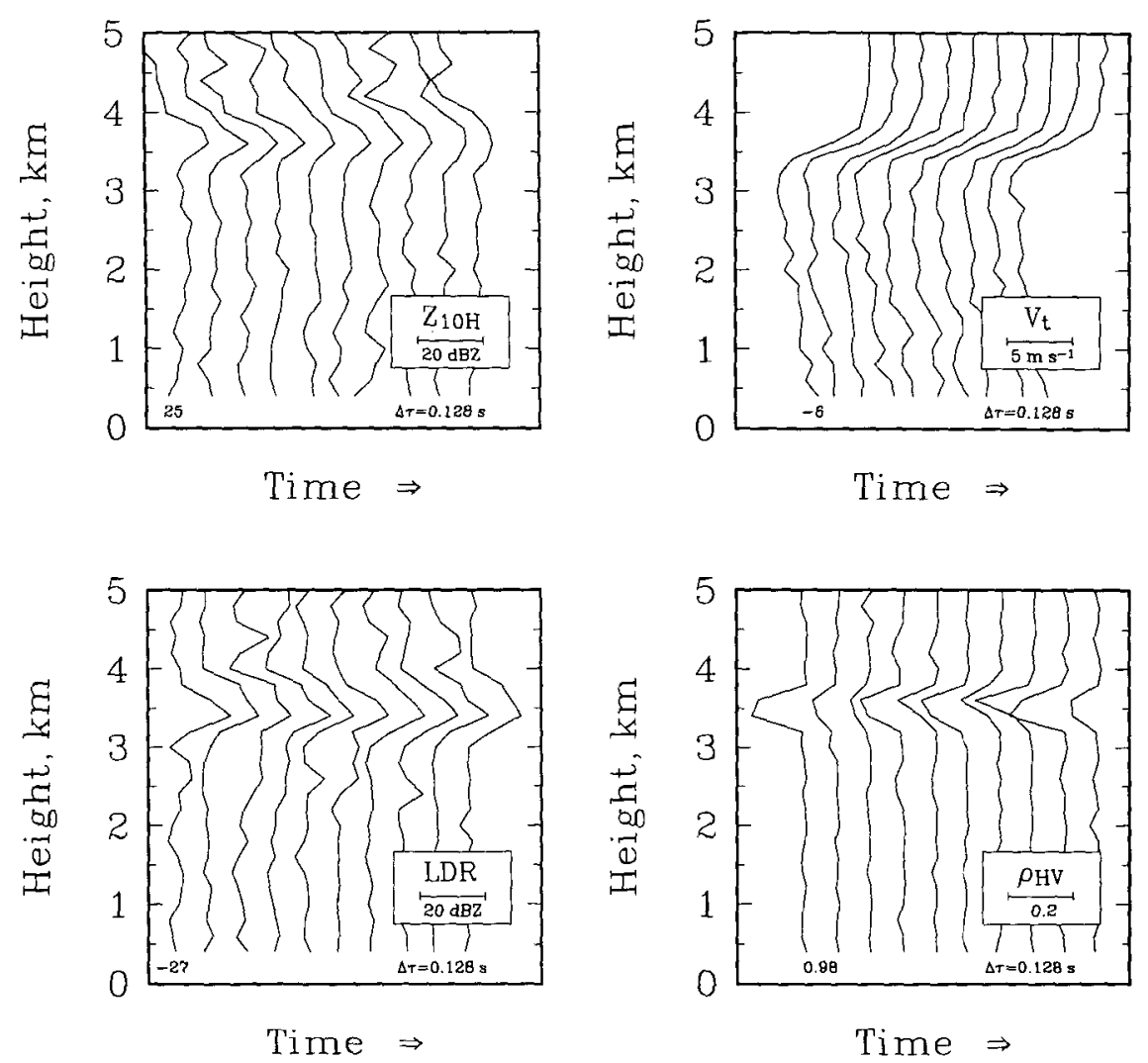

FIG. 3. Vertical profiles of polarimetric variables from CAPE obtained on 25 September 1991. Gate spacing is $200 \mathrm{~m}$, and on this day the $0^{\circ} \mathrm{C}$ isotherm was at $4 \mathrm{~km}$. The linear depolarization ratio LDR is from the $3-\mathrm{cm}$ wavelength radar, which is on the same pedestal as the $10-\mathrm{cm}$ radar. The reflectivity factor, Doppler velocity, and correlation coefficient are from the $10-\mathrm{cm}$ wavelength radar.

The enlarged profiles in Fig. 4e illustrate the relationships between the significant changes of $\left|\rho_{h v}(0)\right|$, $Z$, and $v$ through the melting layer. The change of the velocity from -1 to $-7 \mathrm{~m} \mathrm{~s}^{-1}$ (Fig. $4 \mathrm{c}$ ) occurs between 3.45 and $2.85 \mathrm{~km}$ and coincides with the decrease of the correlation coefficient (Fig. 4e). Over the same height interval there is an increase of the spectrum width (from 1 to $3 \mathrm{~m} \mathrm{~s}^{-1}$ in Fig. 4d). Obviously, the relative amounts of liquid and ice precipitation change within this interval from predominantly ice to rain. The location of the dip in the $\left|\rho_{h v}(0)\right|$ (Fig. 4e) might be where a combined effect of irregular, highly reflective wet aggregates and a highly diverse particle population at various stages of melting is strongest. At the first range location below the dip the correlation coefficient has not yet fully recovered, indicating that large aggregates may be present, in addition to drops, within the resolution volume. Note that a smaller number of large aggregates or irregular drops (with ice cores) is sufficient to reduce the correlation even if the number of spherical small drops is high because the contributions to the correlation coefficient are weighted by the scatterer's cross section.
There were no in situ measurements on this day, but a quick consistency check of radar variables can be made using equations that relate the terminal velocity and the spectrum width to the reflectivity (Atlas et al. 1973). Thus, we use $v_{t}=2.6 Z^{0.107}$, and $\sigma_{v}=0.3 v_{t}$, and apply these to rain below the melting layer; there $Z=3000 \mathrm{~mm}^{6} \mathrm{~m}^{-3}$ (Fig. 4b) from which $v_{t}=6.1$ $\mathrm{m} \mathrm{s}^{-1}$. This is very close to the measured Doppler velocity change of about $-6 \mathrm{~m} \mathrm{~s}^{-1}$ (Fig. 4c). Drops with a $1.8-\mathrm{mm}$ average diameter have a terminal velocity of $6 \mathrm{~m} \mathrm{~s}^{-1}$. Calculation of the spectrum width yields $1.8 \mathrm{~m} \mathrm{~s}^{-1}$, which is smaller than the measured $3 \mathrm{~m} \mathrm{~s}^{-1}$ (Fig. 4d); this is not surprising considering that biases by turbulence, etc., have not been removed from the measured data.

The $Z$ and $\left|\rho_{h v}(0)\right|$ profiles at vertical incidence are very similar to profiles obtained at low elevation angles in the mesoscale convective system of 3 June 1989 (Zrnic et al. 1993). Note that the sharp decrease of $\left\{\rho_{h v}(0)\right\}$ occurs at a single range location (Fig. 4e), implying that the vertical extent of this change is about $150 \mathrm{~m}$; similar thickness was deduced for the June 1989 MCS. Above and below the sharp decrease, the cor- 

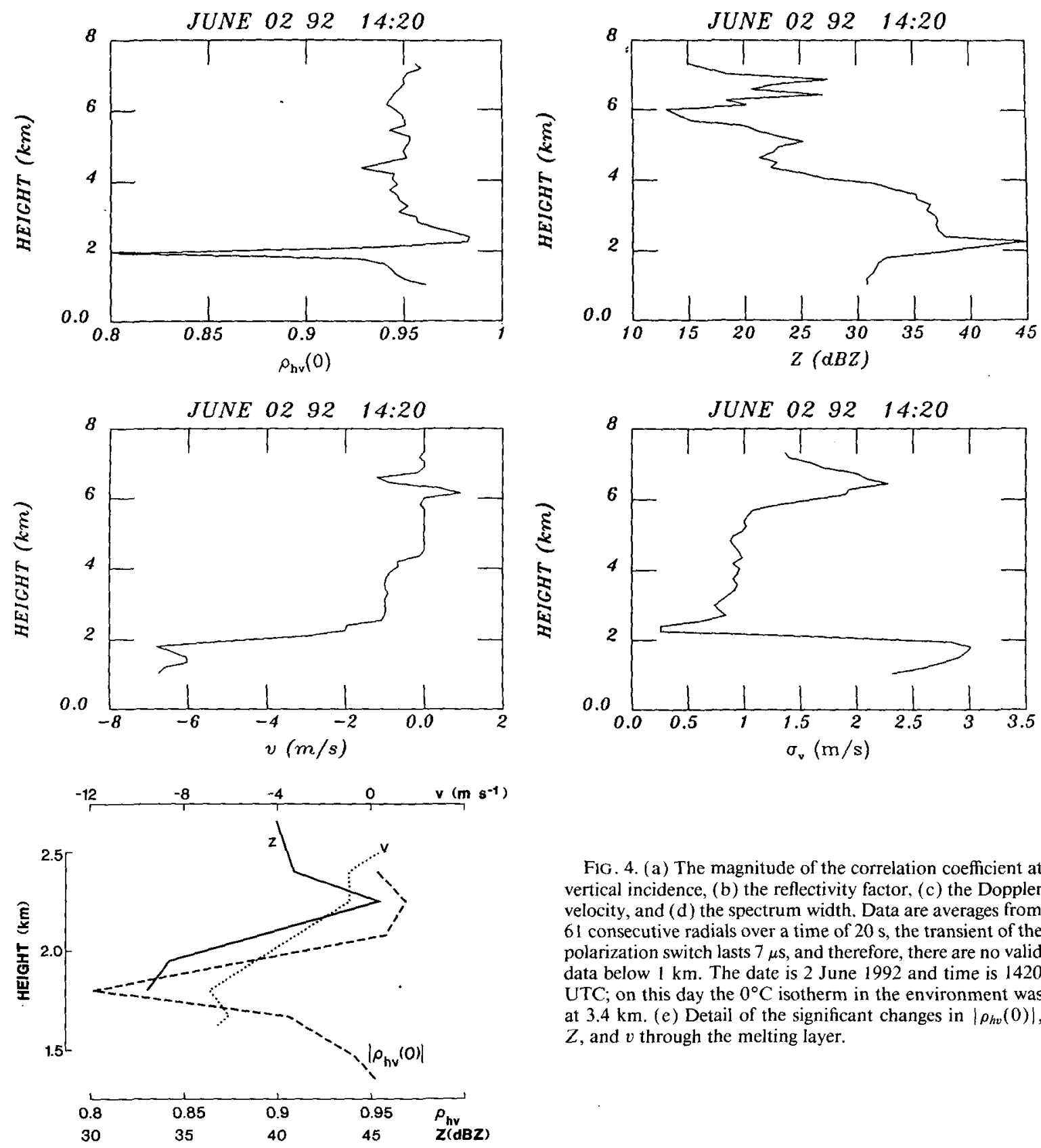

Fig. 4. (a) The magnitude of the correlation coefficient at vertical incidence, (b) the reflectivity factor, (c) the Doppler velocity, and (d) the spectrum width. Data are averages from 61 consecutive radials over a time of $20 \mathrm{~s}$, the transient of the polarization switch lasts $7 \mu \mathrm{s}$, and therefore, there are no valid data below $1 \mathrm{~km}$. The date is 2 June 1992 and time is 1420 UTC; on this day the $0^{\circ} \mathrm{C}$ isotherm in the environment was at $3.4 \mathrm{~km}$. (e) Detail of the significant changes in $\left|\rho_{h v}(0)\right|$, $Z$, and $v$ through the melting layer.

relation is about 0.95 ; this is lower than expected from rain or ice hydrometeors and is attributed to noise bias that could not be completely removed. Otherwise there is not enough information to infer anything else about the significance of the structure in the profile.

Examination of $\left|\rho_{h v}(0)\right|$ at 800 consecutive radials (Fig. 5) confirms that changes are due to evolution and advection. At the beginning of the record the decrease of correlation is at a slightly larger height than at the end but below the location of the melting-layer bottom, which is expected from the sounding.

The changes of the velocity profiles through the melting layer (Figs. 3b and $4 \mathrm{c}$ ) are almost identical in

both cases, which suggests that raindrops below the melting layer had a similar size distribution.

Although it is not known if analogous mechanisms were responsible for the decrease of $\left|\rho_{h v}(0)\right|$, the minimum for both cases is about 0.8 . Illingworth and Caylor (1991) observed with a horizontally pointing beam a mean value of 0.83 , whereas Zrnić et al. (1993) report 0.85 . The current lower values of 0.8 might be negatively biased by quantization noise and artifacts (other than the receiver noise), which cannot be reliably estimated. On the other hand 0.8 is not unusually low considering that the lowest measured value by Illingworth and Caylor (1989) was 0.6. 


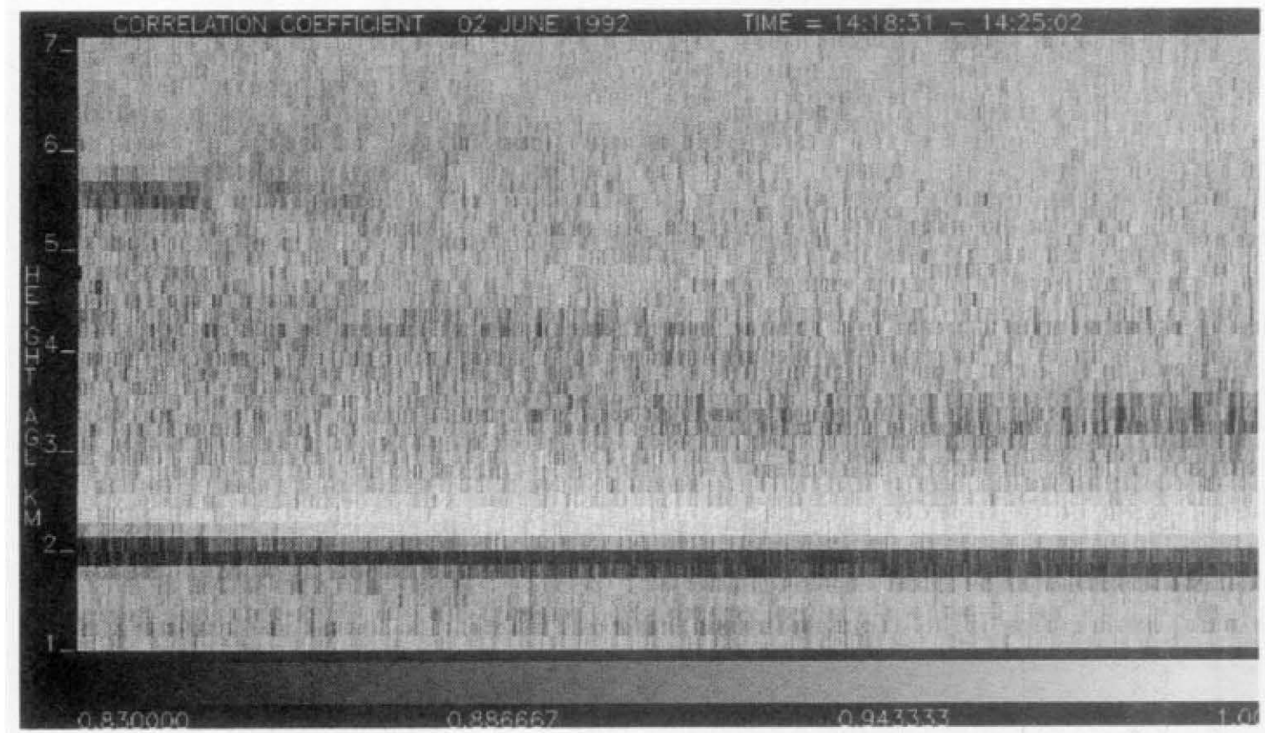

FIG. 5. Time-height cross section of the correlation coefficient for 2 June 1992. The graph contains 800 radials collected during a $320-\mathrm{s}$ time interval; 61 radials starting from the $200 \mathrm{th}$ radial were averaged to obtain the data in Fig. 4.

One of the contributing causes to the decrease of correlation at horizontal incidence is the rapid variation of differential phase shift upon scattering with change in aggregate dimensions. It is not necessary to invoke this Mie effect to explain the decrease of $\left\{\rho_{h v}(0) \mid\right.$ at vertical incidence. Comparison with the two simple models in section 2 suggests that randomly oriented wet prolate spheroids (Fig. 2) with an axis ratio of about 3 would produce a correlation coefficient of 0.8 . Similar value is obtained from wet distorted spheres with an $\mathrm{rms}$ distortion to diameter ratio of 0.15 . From this we construe that hydrometeors have at least this much variation in shape at the bottom of the melting layer.

\section{Summary}

Theoretical and experimental evidence points out a useful property of the correlation coefficient between horizontally and vertically polarized echoes that is obtainable with vertically looking radars. Further, $\left|\rho_{h v}(0)\right|$ provides sharp signatures of the brightband bottom. Precipitation below the melting level consists of a varying mixture of hydrometeors with diverse shape, size, and thermodynamic phase. The presence of such mixtures can result in an observable decrease of $\left|\rho_{h v}(0)\right|$ at vertical incidence. The decorrelation arises from melting, presumably of aggregates, and can be explained in terms of two simple models: one is random oriented wet prolate spheroids and the other is distorted spheres. Prolates with axis ratios of 3 or distorted spheres with rms roughness equal to $15 \%$ of the diameter decrease the correlation to 0.8 , which is the observed minimum at the brightband bottom.
The vertical extent of the $\left|\rho_{h v}(0)\right|$ minima is a few hundred meters and in one case was less then the resolvable length of the measurement $(150 \mathrm{~m})$. Precipitation immediately below the minima is rain and that is deduced from distinct Doppler shift caused by the terminal velocities of drops. Collocated with the Doppler shift is an abrupt change in the spectrum width that reflects the spread of terminal velocities. Because these two changes coincide with the $\left|\rho_{h v}(0)\right|$ minima, we conclude that large irregular aggregates, their collapse into drops, and breakup of big drops are the most likely reasons for the observed signatures.

A desire to locate the bottom of the bright band is not a prime motivating factor behind this research. There are simpler techniques to locate the bright band. For example, the transition between ice and liquid precipitation can be found by observing the change in the mean Doppler velocity or spectrum width. But the correlation coefficient might provide discriminating signatures of hydrometeors, which is not possible with use of the spectral moments. Indications are that different ice crystals should cause a distinct decrease in the correlation coefficient, but there are no in situ measurements to confirm this hypothesis. Independent verification is crucially important for data interpretation and confidence in the polarimetric variables.

We have presented evidence of coherent structures in the time-height display of the correlation coefficient but have not made a detailed study of their significance.

Estimation of the correlation coefficient is fairly simple; furthermore, $\left|\rho_{h v}(0)\right|$ has an advantage over the linear depolarization ratio because it involves measurement of two strong signals as opposed to strong 
and weak signals needed for the linear depolarization ratio.

Acknowledgments. A. Zahrai has contributed substantially to the upgrade of the Cimarron radar facility. M. Schmidt has made numerous design changes and modifications of various hardware; he is also maintaining the facility with the help of $G$. Anderson and R. Wahkinney. Continuous collaboration with N. Balakrishnan (Indian Institute of Science, Bangalore, India) lead to insights into the interpretation of polarimetric data. Yosepha Gal-Chen and Brent Gordon generated several figures for this paper; other graphs were produced by Joan Kimpel. T. Matejka and V. Mazur provided useful suggestions concerning the manuscript. A. Jameson thoroughly reviewed the manuscript and was instrumental in arranging collaboration between the authors. We appreciate interaction with the Operational Support Facility, NWS, and the funding provided by the FAA for much of the receiver hardware. This research was supported in part by the NASA Order Number S-64075-E.

\section{REFERENCES}

Antar, A. M. M., and A. A. Hendry, 1985: Correlation measurements in precipitation at linear polarization using dual-channel radar. Electron. Lett., 21, 1052-1054.

Atlas, D., R. C. Srivastava, and R. S. Sekhon, 1973: Doppler radar characteristics of precipitation at vertical incidence. Rev. Geophys. Space Phys, 2, 1-35.

Aydin, K., T. A. Seliga, and V. Balaji, 1986: Remote sensing of hail with a dual linear polarization radar. J. Climate Appl. Meteor., $25,1475-1484$.

Balakrishnan, N., and D. S. Zrnić, 1990: Use of polarization to characterize precipitation and discriminate large hail. J. Atmos. Sci., 47, 1525-1540.

Battan, L. J., 1973: Radar Observation of the Atmosphere. University of Chicago Press, 324 pp.

Bringi, V. N., V. Chandrasekar, P. Meischner, J. Hubbert, and Y. Golestani, 1991: Polarimetric radar signatures of precipitation at $\mathrm{S}$ - and C-bands. IEEE Proc. $F, 138,109-119$.

Doviak, R. J., and D. S. Zrnić, 1993: Doppler Radar and Weather Observations. Academic Press, 562 pp.

Hendry, A., and G. C. McCormick, 1976: Radar observations of the alignment of precipitation particles by electrostatic fields in thunderstorms. J. Geophys. Res., 81, 5353-5357.

,-- , and L. G. Barge, 1976: The degree of common orientation of hydrometeors observed by polarization diversity radar. $J$. Appl. Meteor., 15, 633-640.
Illingworth, A. J, and I. J. Caylor, 1989: Cross polar observations of the bright band. Preprints, 24th Conf. on Radar Meteorology, Tallahassee, FL, Amer. Meteor. Soc., 323-327.

- , and,- 1991 : Co-polar measurement of precipitation. Preprints, 25th Conf. on Radar Meteorology, Paris, Amer. Meteor. Soc., 650-653.

Jameson, A. R., 1987: Relations among linear and circular polarization parameters measured in canted hydrometeors. J. Atmos. Oceanic Technol., 4, 634-645.

__, 1989a: The interpretation and meteorological application of radar backscatter amplitude ratios at linear polarizations. $J$. Atmos. Oceanic Technol., 6, 908-919.

- , 1989b: The application of radar backscatter amplitude ratios at linear polarizations to precipitation studies. Preprints, URSI Open Symp., La Londes-les Maures, 2.3.1-2.3.4.

- - and J. H. Dave, 1988: An interpretation of circular polarization measurements affected by propagation differential phase shift. J. Atmos. Oceanic Technol., 5, 405-415.

Liu, L., V. N. Bringi, V. Chandrasekar, E. A. Mueller, and A. Muducutore, 1993: Statistical characteristics of the copolar correlation coefficient between horizontal and vertical polarizations. Preprints, 26th Int. Conf. on Radar Meteorology, Norman, OK, Amer. Meteor. Soc., 115-119.

Metcalf, J. I., 1993: Observation of the effects of changing electric fields on the orientation of hydrometeors in a thunderstorm. Bull. Amer. Meteor. Soc., 74, 1080-1093.

McCormick, G. C., and A. Hendry, 1985: Optimal polarizations for partially polarized backscatter. IEEE Trans. Antennas Propag., AP-3, 33-40.

Sachidananda, M., and D. S. Zrnić, 1985: $Z_{\mathrm{DR}}$ measurement considerations for a fast scan capability radar. Radio $S_{c i}$. 20, $907-$ 922.

$\ldots$, and _ $\ldots$, 1986: Differential propagation phase shift and rainfall rate estimation. Radio $S c i, 21,235-247$.

- $\ldots$, and - 1987 : Rain rate estimates from differential polarization measurements. J. Atmos. Oceanic Technol., 4, 588-598.

Seliga, T. A., and V. N. Bringi, 1978: Differential reflectivity and differential phase shift: Applications in radar meteorology. Radio Sci., 13, 271-275.

Ulbrich, C. W., and D. Atlas, 1984: Assessment of the contribution of differential polarization to improved rainfall measurements. Radio Sci., 19, 49-57.

Willis, T. W., and A. J. Heymsfield, 1989: Structure of the melting layer in mesoscale convective system stratiform precipitation. J. Atmos. Sci., 46, 2008-2025.

Zahrai, A., and D. S. Zrnic, 1993: The 10-cm wavelength polarimetric weather radar at NOAA's National Severe Storms Laboratory. J. Atmos, Oceanic Technol., 10, 649-662.

Zrnić, D. S., 1991: Complete polarimetric and Doppler measurements with a single receiver radar. J. Atmos. Oceanic Technol., 8, 159165.

—, N. Balakrishnan, C. L. Ziegler, V. N. Bringi, K. Aydin, and T. Matejka, 1993: Polarimetric signatures in the stratiform region of a mesoscale convective system. J. Appl. Meteor., 32, 678693. 